\section{Corneal astigmatism in Graves' ophthalmopathy}

\author{
Abstract \\ Purpose To describe corneal astigmatism in \\ patients under the age of 55 years with Graves' \\ ophthalmopathy and to compare it to a control \\ group. \\ Methods Retrospective, nonrandomized \\ comparative observational case series. \\ A total of 109 patients with inactive \\ Graves' ophthalmopathy and 109 age- \\ and gender-matched control individuals \\ without Graves' ophthalmopathy were \\ examined with manual corneal keratometry. \\ In the Graves' patients, $52 \%$ had a CT \\ scan available for review, and $41 \%$ \\ underwent orbital, strabismus, and/or \\ eyelid surgery with a follow-up of \\ keratometry over a mean period of 1.7 years \\ after surgery. \\ Results For the dioptres, there was a \\ statistically significant difference between \\ the Graves' and the control eyes at 3-3.25 \\ dioptres in both eyes ( $t$-test, right eyes, \\ $P=0.042$; left eyes, $P=0.041$ ). For the \\ meridians, the astigmatism was found to be \\ greater at the meridians $95-100^{\circ}$ ( $t$-test, \\ $P=0.044)$ and $105^{\circ}-110^{\circ}(t$-test, $P=0.017)$ \\ in the right eyes only. In $10(9 \%)$ Graves' $^{\prime}$ \\ patients, the astigmatism had caused \\ newly acquired reduced visual acuity. \\ Greater astigmatism in dioptres did not \\ correlate with specific CT scan findings $\left(\chi^{2}\right.$-test, \\ $\mathrm{df}=2, P=0.187$ ). Following orbital, \\ strabismus, and eyelid surgery, the dioptres \\ and meridians of the astigmatism did not \\ change in $56(68 \%)$ and $66(81 \%)$ eyes, \\ respectively. \\ Conclusion Graves' ophthalmopathy may be \\ associated with greater with-the-rule corneal \\ astigmatism, which, overall, is not influenced \\ by orbital, strabismus, or eyelid surgery. The \\ astigmatism may possibly be caused by soft- \\ tissue fibrosis in the superolateral orbital \\ region. \\ Eye (2006) 20, 440-446. doi:10.1038/sj.eye.6701898; \\ published online 22 April 2005
}

I Mombaerts, S Vandelanotte and L Koornneef

Keywords: corneal astigmatism; Graves' ophthalmopathy; keratometry; orbit; cornea; visual acuity

\section{Introduction}

Many patients with Graves' ophthalmopathy complain of reduction in vision, ${ }^{1}$ possibly caused by corneal exposure keratopathy or optic nerve compression. ${ }^{2,3}$ We noticed the prevalence of corneal astigmatism in a subset of patients with Graves' ophthalmopathy as another cause of blurred vision. To our knowledge, neither the presence nor the pattern of corneal astigmatic errors in Graves' ophthalmopathy have been extensively reported in the literature, except for a few case reports on acquired keratoconus in Graves' ophthalmopathy. 4,5 The present study retrospectively analyses the keratometry data of Graves' ophthalmopathy patients. In order to identify the possible causal factors of astigmatism, keratometry data were followed after orbital, strabismus, and eyelid surgery.

The normal cornea has a curvature, characterized by a physiological with-the-rule astigmatism. ${ }^{6}$ Epidemiological studies on corneal astigmatism unfortunately do not provide detailed information on the dioptres and meridians of astigmatism in specific age and gender groups. ${ }^{7,8}$ For that reason, we included in our study an age- and gendermatched control group of individuals without Graves' ophthalmopathy.

\section{Materials and methods}

The medical records, clinical photographs, and CT scans of a group of patients with the established diagnosis of Graves' ophthalmopathy were analysed retrospectively. All patients were consecutively seen at the Orbital Clinic of the University Hospital Leuven and were examined by one of the authors (IM). 
The diagnosis of Graves' ophthalmopathy was based on one or more of the clinical findings of bilateral or unilateral upper eyelid retraction, proptosis, extraocular muscle restriction, and/or the CT scan finding of rectus muscle enlargement in patients with present or past serological evidence for thyroid disease. Corneal keratometry readings were used when Graves' ophthalmopathy had been stable and inactive for at least 6 consecutive months. ${ }^{9}$

The control individuals were selected on age and gender to match the distribution of the Graves' patients. They were healthy volunteers and patients without Graves' ophthalmopathy referred to the general eye clinic for routine eye control on the basis of headache, systemic hypertension, diabetes mellitus, or presbyopia. They were recruited over a period of 6 months.

All individuals older than 55 years were excluded for reasons of corneal astigmatic changes related to aging. ${ }^{7,8,10}$ Excluded were all Graves' and control eyes with previous eye, eyelid, strabismus, or orbital surgery. Because of the increased incidence of astigmatic errors in eyes with high spherical errors and in amblyopic eyes, all eyes were excluded with 6 dioptres or more of myopia or hypermetropia, and amblyopia. ${ }^{6}$

Central corneal astigmatism was measured by standard manual keratometry, using the same JavalSchiotz keratometer for all exams. A meridian of steepest curvature at $90 \pm 15^{\circ}$ was designated as with-the-rule astigmatism; at $165-15^{\circ}$ (around $0^{\circ}$ ) as against-the-rule astigmatism, and outside of these meridians as oblique astigmatism. ${ }^{7}$ No curvature in the $180^{\circ}$ meridian was assigned as 0 dioptre at $90^{\circ}$. Manual keratometry was performed as a single reading, by the same examiner (IM) at each visit for the Graves' patients and by one of the two examiners (IM, SV) for the control individuals.

In the Graves' patients, assessment of upper eyelid position was made in the primary position of gaze for each individual eye. Upper eyelid retraction was scored on the amount of scleral show in $\mathrm{mm}$, in the 12 o'clock position, from the upper eyelid margin to the limbus. Proptosis was diagnosed when one of the following parameters was present: Hertel exophthalmometer reading of $16 \mathrm{~mm}$ or more, ${ }^{11}$ anteriorly displaced globe on axial CT scan, clinical proptosis when compared to predisease clinical photographs. Assessment of extraocular muscle restriction was made by clinical eye motility testing for each individual eye in all directions of gaze.

Coronal and axial orbital CT scans of unoperated Graves' patients were reviewed for levator palpebrae superioris and rectus muscle enlargement. The lacrimal gland was evaluated on axial scans, and was recorded as displaced if half or more of the lacrimal gland was anterior to the frontozygomatic process, and was labelled as enlarged if the size was abnormally thickened.

Enlargement of the tensor intermuscularis muscle ${ }^{12}$ and fibrosis of the superolateral orbit were assessed on coronal scans. Fibrosis of the superolateral orbit was defined as grey to white areas or clearly visible orbital septa in the area between the levator palpebrae superioris muscle and the lacrimal gland, anterior to the tensor intermuscularis muscle. Excluded were all planes of CT scans with insufficient quality to judge one of these parameters.

The two-sample $t$-test, $\chi^{2}$-test, and linear regression $(R)$ were used for statistical analysis. All $P$-values of the $t$-test were two-sided. Both eyes of the patient were used as unpaired variables because Graves' ophthalmopathy frequently affects the orbits asymmetrically.

\section{Results}

From August 1998 to October 2000, 160 patients with Graves' ophthalmopathy were seen. In total, 51 patients (including six patients with optic neuropathy) were not eligible for the study because of: age over 55 years (31 patients), bilateral high myopia (two patients), bilateral high hypermetropia (one patient), previous eye, eyelid, squint or orbital surgery (five patients), and insufficient data (12 patients). In nine patients, one eye only was studied because of unilateral Graves' ophthalmopathy (eight patients) or contralateral amblyopia (one patient). The Graves' group comprised 109 patients (209 eyes, 107 right and 102 left). There were 87 female and 22 male, all Caucasian except two Asian and one black female. The mean age was 43.5 years (range $=20-55$, median $=45.2$, $95 \%$ confidence interval $(\mathrm{CI})=26.4-60.6, \mathrm{SD}=8.7$, $\mathrm{SE}=0.8)$. In all, 14 patients had received orbital radiotherapy, three patients oral corticotherapy, and three patients a combination of both therapies. None of the patients had optic neuropathy.

The control group consisted of 109 Caucasian individuals of whom 47 healthy volunteers and 62 general eye clinic patients. There were 87 female and 22 male, with a mean age of 42.8 years (range $=20-55$, median $=44.7,95 \% \mathrm{CI}=25.7-59.6, \mathrm{SD}=8.7, \mathrm{SE}=0.8$ ). The age of the controls and Graves' patients was statistically not different ( $t$-test, $P=0.513$ ).

Figure 1 shows the patterns of astigmatism found in the Graves' eyes and control eyes for the right eyes, and Figure 2 for the left eyes. For the dioptres, there was a statistically significant difference between the Graves' and the control eyes at 3-3.25 dioptres in both eyes ( $t$-test, right eyes, $P=0.042$; left eyes, $P=0.041$ ). For the meridians, the astigmatism was found to be greater at the meridians $95-100^{\circ}(t$-test, $P=0.044)$ and $105-110^{\circ}$ ( $t$-test, $P=0.017)$ in the right eyes only. 

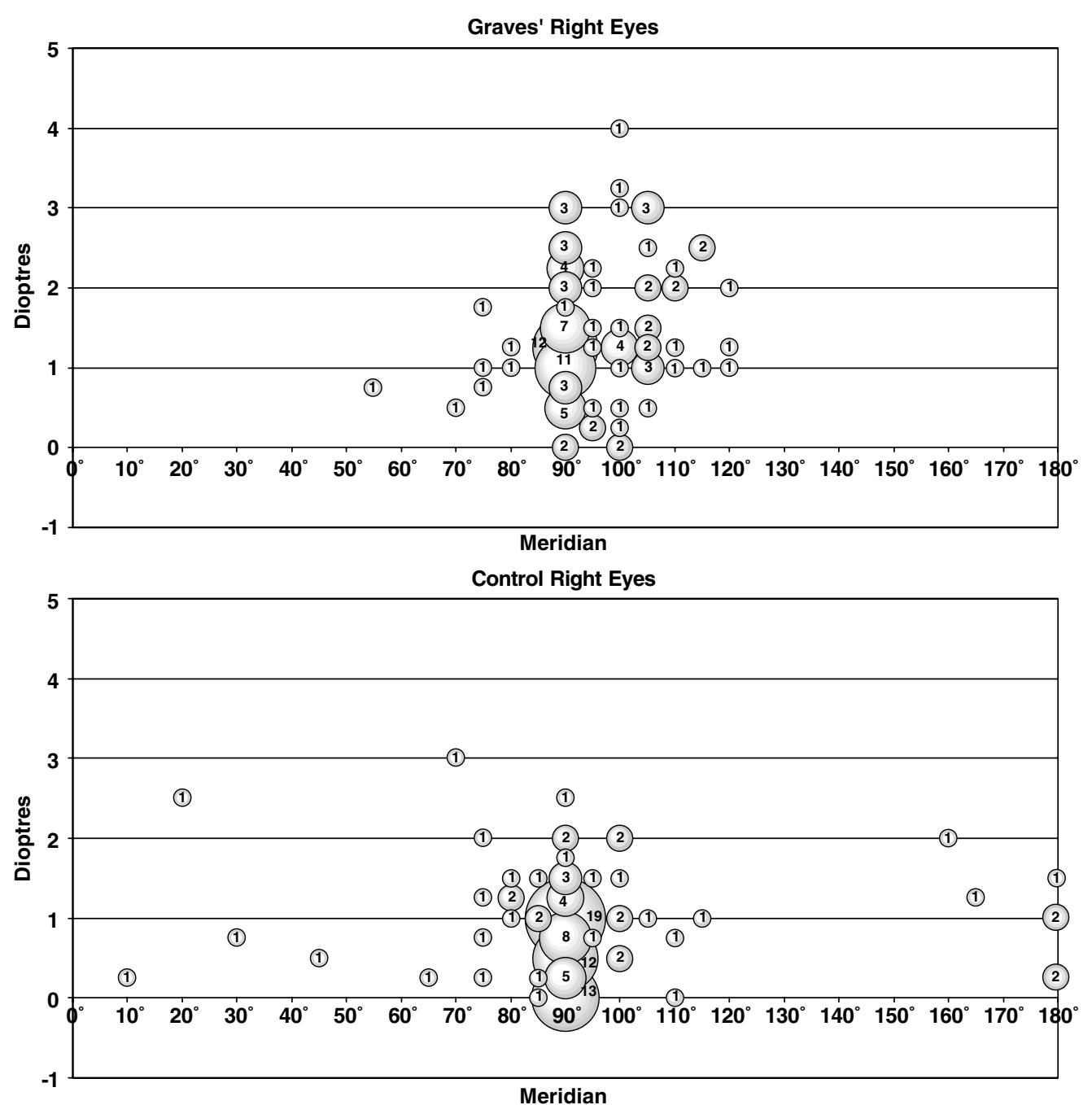

Figure 1 Corneal astigmatic patterns in 107 right eyes with Graves' ophthalmopathy and 107 right eyes without Graves' ophthalmopathy (control). The numbers in and the size of the bubbles represent the number of the eyes.

In $9 \%$ of the Graves' patients (10 patients, 18 eyes) visual acuity was $20 / 30$ or less and could be improved to $20 / 20$ with cylinders with negative power in the horizontal axis. None of these patients did have these cylindric correction glasses or toric contact lenses prior to the onset of Graves' ophthalmopathy.

Clinical parameters of Graves' ophthalmopathy including upper eyelid retraction, proptosis, and eye motility restriction were compared to the degree of corneal astigmatism in dioptres. There was a significant association between astigmatism of more than 1.25 dioptres and the presence of upper eyelid retraction $\left(\chi^{2}=6.67\right.$; degrees of freedom $\left.(\mathrm{df})=2, P=0.036\right)$ but not with the presence of proptosis $\left(\chi^{2}=3.85, \mathrm{df}=2\right.$, $P=0.146)$ and eye motility restriction $\left(\chi^{2}=2.81, \mathrm{df}=2\right.$, $P=0.246)$. The association was found predominantly at the meridian of $90^{\circ}$. The amount of corneal astigmatism in dioptres did not correlate with the amount of upper eyelid retraction in $\mathrm{mm}$ (linear regression, 95\%

$\left.\mathrm{CI}=0.178-0.324, R^{2}=0.181, P<0.0001\right)$ or the amount of proptosis in $\mathrm{mm}$ (linear regression, $95 \% \mathrm{CI}=0.004-0.060$; $\left.R^{2}=0.025 ; P=0.024\right)$.

CT scans were available in 57 patients (52\%). Evident clinical diagnosis of Graves' ophthalmopathy and no need for treatment had not warranted a CT scan in the remaining patients. Enlargement of the levator palpebrae superioris muscle was found in $43 \%$ of the eyes, enlarged inferior rectus muscle in $61 \%$, enlarged medial rectus muscle in $47 \%$, enlarged superior rectus muscle in $41 \%$, enlarged lateral rectus muscle in $28 \%$, displaced lacrimal gland in $34 \%$, enlarged lacrimal gland in $41 \%$, enlarged tensor intermuscularis muscle in $47 \%$, and fibrosis of the superolateral orbit in $74 \%$ (Figure 3). There was no statistical association between astigmatism of more than 1.25 dioptres and any of these CT scan findings ( $\chi^{2}$-test, $\mathrm{df}=2$, lowest value of $P=0.187$ ). 

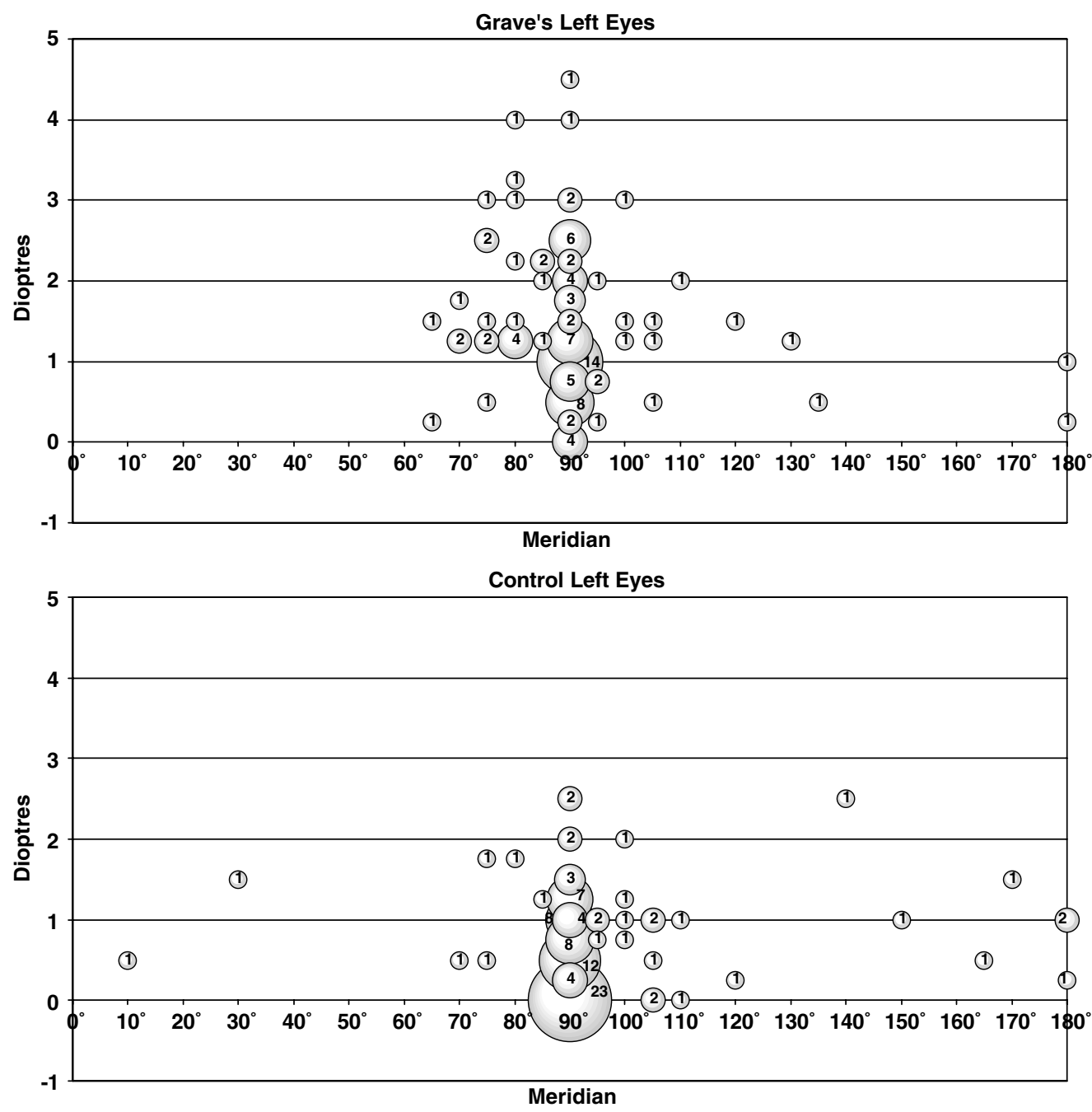

Figure 2 Corneal astigmatic patterns in 102 left eyes with Graves' ophthalmopathy and 102 left eyes without Graves' ophthalmopathy (control). The numbers in and the size of the bubbles represent the number of the eyes.

Totally, 45 Graves' patients (41\%, 82 eyes) had surgery and a keratometric follow-up. Orbital decompression was performed using the coronal approach in 26 patients and the lower eyelid approach in six patients. Strabismus surgery was performed in 49 eyes implying rectus and oblique muscle recessions. In 41 eyes, upper eyelid lengthening was achieved by en bloc recession of the posterior lamella via anterior lid crease incision. ${ }^{13}$ In 14 eyes an upper eyelid blepharoplasty, in nine eyes a lower eyelid lengthening with donor sclera interpositioning, and in four eyes a lower eyelid blepharoplasty was performed. Figure 4 illustrates the astigmatic changes after all surgical procedures compared to the preoperative values in 82 eyes. Any change of 0.50 dioptre or $10^{\circ}$ of meridian was considered insignificant or within range of error of keratometry. Following surgery, increased steepening of more than 0.50 dioptre or a shift of more than $10^{\circ}$ away from the with-the-rule meridian was considered 'worsened.' Decreased steepening of more than 0.50 dioptre or a shift of more than $10^{\circ}$ towards the with-the-rule meridian was considered 'improved.' There was no change of dioptres in 56 eyes $(68 \%)$ and no change of meridians in 66 eyes $(81 \%)$ at the keratometric follow-up (mean $=1.7$ years, median $=1.5$, range $=0.3-4.6, \mathrm{SD}=1.1$ ). When looking at each surgical intervention separately, after orbital decompression no change in dioptres and meridian was observed in 38 eyes (73\%) and 41 eyes (79\%), respectively, after strabismus surgery in 21 eyes $(68 \%)$ and 27 eyes (87\%), after upper eyelid lengthening in 31 eyes $(78 \%)$ and 35 eyes $(88 \%)$, and after upper eyelid blepharoplasty in eight eyes (67\%) and 11 eyes (92\%). The worsened and improved astigmatic changes after each surgical procedure are similarly distributed as in Figure 4. The mean keratometric follow-up after orbital decompression was 5 months, after strabismus surgery 
a
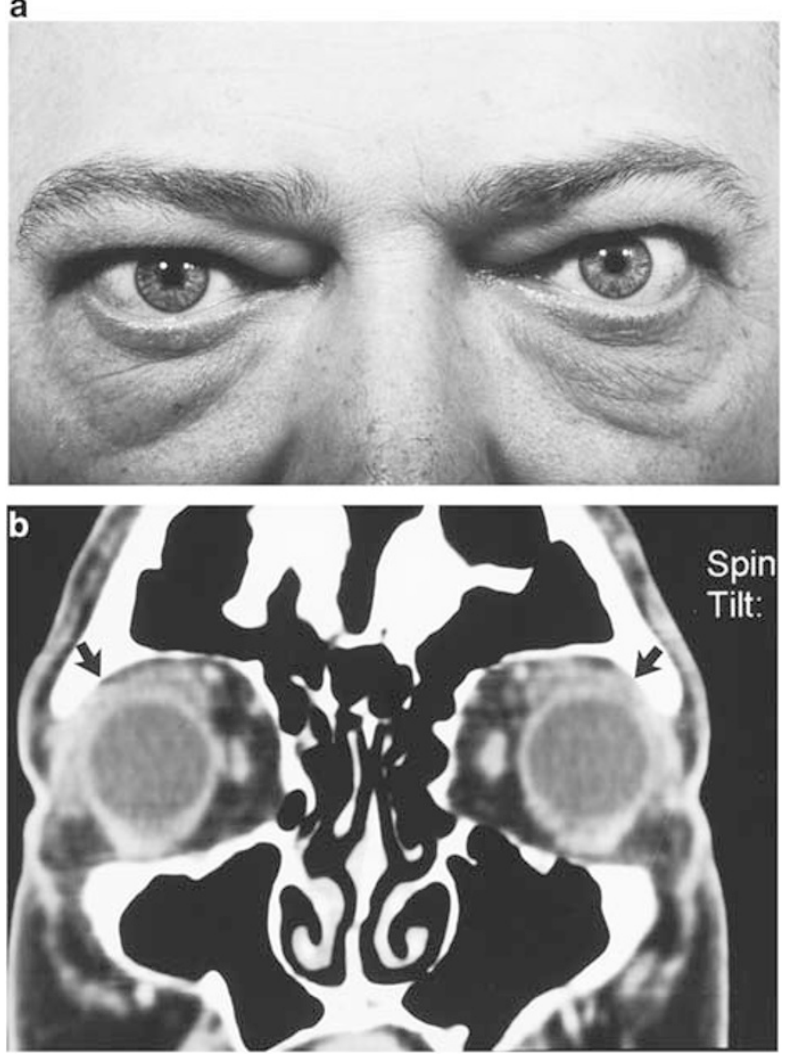

Figure 3 (a) Clinical photograph and (b) orbital CT scan coronal view of a 46-year-old male patient with Graves' ophthalmopathy. He had bilateral upper eyelid retraction, proptosis, and eye motility restriction in abduction. The manual keratometry reading of the right eye was 2.25 dioptres steepening in the $95^{\circ}$ meridian, and of the left eye 2.25 dioptres steepening in the $75^{\circ}$ meridian. Fibrosis in the superolateral orbit is seen (arrows).

11 months, after upper eyelid lengthening 13 months, and after upper eyelid blepharoplasty 6 months.

\section{Discussion}

The eyes of patients with inactive unoperated Graves' ophthalmopathy demonstrated a statistically significant greater steepening of the central cornea in the with-therule meridian compared to the control eyes. As a referral centre, we recognize that our Graves' group may represent a selected population. But $48 \%$ of the patients did not warrant a CT scan mainly because of the mild nature of their Graves' ophthalmopathy. In order not to bias our population of Graves' ophthalmopathy, we included the patients without CT scan in the present study. Consequently, the results from the CT scan review are skewed and should be interpreted as such.

A drawback of the study is that the corneal curvature was measured with manual keratometry and not with

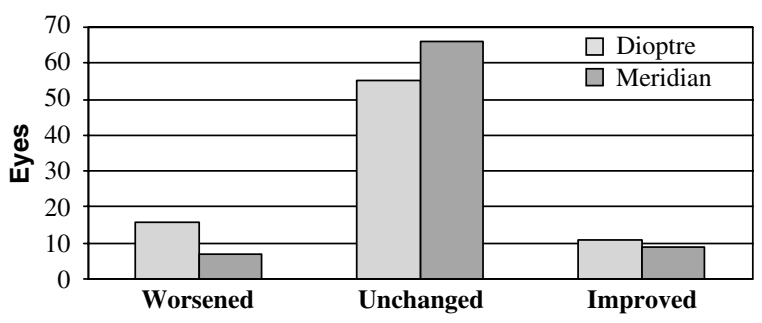

Figure 4 Corneal astigmatic changes of 82 Graves' eyes following all surgical procedures compared to the preoperative values. The changes of dioptres and meridians are shown separately. Worsened is defined as increased steepening of more than 0.50 dioptre or a shift of more than $10^{\circ}$ away from the withthe-rule meridian. Improved is defined as decreased steepening of more than 0.50 dioptre or a shift of more than $10^{\circ}$ towards the with-the-rule meridian.

computerized corneal topography. The latter has the advantages of objective measuring and additionally examining the peripheral corneal curvature. However, all keratometry readings in the Graves' patients were performed by the same examiner, so that interobserver differences were eliminated.

Essentially, three pathologic processes can produce abnormal astigmatism of the cornea. These include abnormalities of the corneal epithelium, alteration in stromal thickness, and external compression by masses. ${ }^{14,15}$ Upper eyelid swelling may act like a mass. Its removal led to increased with-the-rule astigmatism in a study of Brown et al ${ }^{16}$ on patients without Graves' ophthalmopathy shortly after upper eyelid blepharoplasty. Yet, our study disclosed no astigmatic change in many of the Graves' eyes 6 months after upper eyelid blepharoplasty. Therefore, the mass effect from upper eyelid swelling probably is not the cause of astigmatism in Graves' ophthalmopathy.

Eyelids exert pressure on the cornea by tension and position. ${ }^{17}$ Upper eyelid tension decreases with age and is presumably the cause of the corneal astigmatic change from predominantly with-the-rule in the young to more oblique and against-the-rule in older subjects. ${ }^{10}$ Likewise, experiments with lifting the eyelids from the surface of the cornea demonstrate a shift of the corneal curvature in the direction of less with-the-rule astigmatism. ${ }^{18,19}$ Our study did show evidence of correlation between greater astigmatism in dioptres and the presence of upper eyelid retraction. However, in most of the eyes no astigmatic shift was observed following restoring the upper eyelid position. Therefore, upper eyelid retraction itself presumably does not produce corneal astigmatism in Graves' ophthalmopathy.

Another plausible mechanism for corneal astigmatism may be forces transmitted to the cornea via the sclera derived from extraocular muscle tension. In a 
computerized corneal topography analysis study of five patients with Graves' ophthalmopathy, Kwitko et $a l^{20}$ found a flattening of the superior and a steepening of the inferior peripheral cornea 1 month following inferior rectus muscle recession. In the present study, 11 months after strabismus surgery, a high percentage of the eyes did not show a shift of dioptres or meridian in the central cornea. Therefore, tethering effect from restricted extraocular muscles may be negligible in the origin of corneal astigmatism in Graves' ophthalmopathy.

We suggest that other structures exert local indentation of the globe in Graves' ophthalmopathy. As astigmatism was found in the with-the-rule meridian with a tendency towards the temporal oblique meridian, attention was

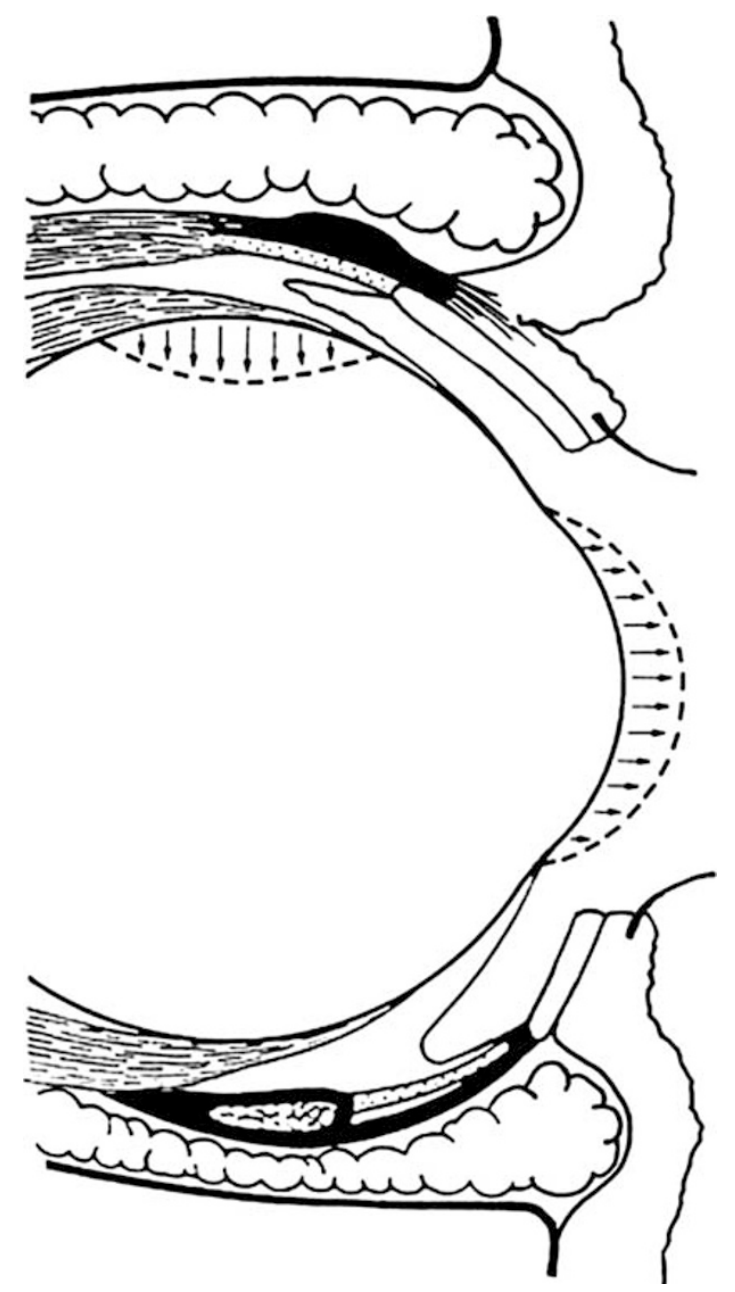

Figure 5 Schematic drawing of a sagittal view of the superolateral region of the orbit, for the proposed hypothesis for greater with-the-rule astigmatism in Graves' ophthalmopathy. Fibrosis of orbital structures in the superolateral region causes indentation of the sclera at the equator of the globe (arrows and dotted line above) resulting in secondary steepening of the cornea in the with-the-rule meridian (arrows and dotted line right). focused on the superolateral orbital region. However, a radiographically enlarged levator/superior rectus / lateral rectus muscle or lacrimal gland or tensor intermuscularis muscle did not correlate with greater astigmatism. In Graves' ophthalmopathy, the superolateral orbit is more severely affected than the superomedial because of the more developed connective tissue system in the area between the levator palpebrae superioris muscle and the lacrimal gland, which is a clinically well-known observation during upper eyelid lengthening surgery. ${ }^{21,22} \mathrm{~A}$ possible mechanism for the astigmatism is that soft-tissue fibrosis in the superolateral orbital region is applying pressure on the sclera at the equator of the globe, resulting in a secondary steepening of the cornea in the with-the-rule meridian (Figure 5). Our CT scan review yielded no correlation between astigmatism and fibrosis in the superolateral orbit. In fact, we found it most difficult to judge fibrosis in the superolateral orbit on CT scan because of the crowding of anatomic structures in the area between the frontal bone and the globe.

In conclusion, Graves' ophthalmopathy may be associated with greater with-the-rule astigmatism overall not influenced by orbital, strabismus, or eyelid surgery. In the absence of other, proven, causal factors, we suggest fibrosis in the superolateral orbit as the possible cause.

\section{Acknowledgements}

We thank Lidwine Van Malderen, Eng, Department of Ophthalmology, University Hospital Leuven and Michael P Hopwood, BSc, for the statistical and mathemathical assistance.

\section{References}

1 Terwee CB, Gerding MN, Dekker FW, Prummel MF, Wiersinga WM. Development of a disease-specific quality of life questionnaire for patients with Graves' ophthalmopathy: The GO-QOL. Br J Ophthalmol 1998; 82: 773-779.

2 Khurana AK, Sunder S, Ahluwalia BK, Malhotra KC. Tear film profile in Graves' ophthalmopathy. Acta Ophthalmol 1992; 70: 346-349.

3 Trobe JD. Optic nerve involvement in dysthyroidism. Ophthalmology 1981; 88: 488-492.

4 King EF. Keratoconus following thyroidectomy. Trans Ophthalmol Soc UK 1953; 73: 31-39.

5 Jacobs DS, Green WR, Maumenee AE. Acquired keratoglobus. Am J Ophthalmol 1974; 77: 393-399.

6 Duke-Elder S, Abrams D. Anomalies of refraction and accommodation. In: Duke-Elder S (ed). System of Ophthalmology, Volume V: Ophthalmic Optics and Refraction. Henry Kimpton: London, 1970, pp 274-283.

7 Fledelius HC, Stubgaard M. Changes in refraction and corneal curvature during growth and adult life. Acta Ophthalmol 1986; 64: 487-491. 
8 Leighton DA, Tomlinson A. Changes in axial length and other dimensions of the eyeball with increasing age. Acta Ophthalmol 1972; 50: 815-826.

9 Mourits MP, Koornneef L, Wiersinga WM, Prummel MF, Berghout A, van der Gaag R. Clinical criteria for the assessment of disease activity in Graves' ophthalmopathy: a novel approach. Br J Ophthalmol 1989; 73: 639-644.

10 Gudmundsdottir E, Jonasson F, Jonsson V, Stefánsson E, Sasaki H, Sasaki K. 'With the rule' astigmatism is not the rule in the elderly. Acta Ophthalmol (Scand) 2000; 78: 642-646.

11 Migliori ME, Gladstone GJ. Determination of the normal range of exophthalmometric values for black and white adults. Am J Ophthalmol 1984; 98: 438-442.

12 Goodall KL, Jackson A, Leatherbarrow B, Whithouse RW. Enlargement of the tensor intermuscularis muscle in Graves' ophthalmopathy. Arch Ophthalmol 1995; 113: 1286-1289.

13 Mombaerts I. The upper eyelid in Graves' ophthalmopathy. Oper Techn Oculoplastic Orbital Reconstr Surg 1999; 2: 42-46.

14 Plager DA, Snyder SK. Resolution of astigmatism after surgical resection of capillary hemangiomas in infants. Ophthalmology 1997; 104: 1102-1106.

15 Troutman RC, Buzard KA. Anatomy and physiology. In: Troutman RC, Buzard KA (eds). Corneal Astigmatism:
Etiology, Prevention, and Management. Mosby Year Book: St Louis, MO, 1992, pp 23-35.

16 Brown MS, Siegel IM, Lisman RD. Prospective analysis of changes in corneal topography after upper eyelid surgery. Ophthalmic Plast Reconstr Surg 1999; 15: 378-383.

17 Vihlen FS, Wilson G. The relation between eyelid tension, corneal toricity, and age. Invest Ophthalmol Vis Sci 1983; 24: 1367-1373.

18 Wilson G, Bell C, Chotai S. The effect of lifting the lids on corneal astigmatism. Am J Optom Physiol Optics 1982; 59: 670-674.

19 Cuttone JM, Durso F, Miller M, Evans LS. The relationship between soft tissue anomalies around the orbit and globe and astigmatic refractive errors: a preliminary report. J Pediatr Ophthalmol Strabismus 1980; 17: 29-36.

20 Kwitko S, Feldon S, McDonnell PJ. Corneal topographic changes following strabismus surgery in Graves' disease. Cornea 1992; 11: 36-40.

21 Koornneef L. Eyelid and orbital fascial attachments and their clinical significance. Eye 1988; 2: 130-134.

22 Ettl A, Priglinger S, Kramer J, Koornneef L. Functional anatomy of the levator palpebrae superioris muscle and its connective tissue system. Br J Ophthalmol 1996; 80: 702-707. 